\title{
Influence of a dense photosphere-like layer on vertical oscillations of a curved coronal slab
}

\author{
M. Gruszecki ${ }^{1}$, K. Murawski ${ }^{1}$, and J. A. McLaughlin ${ }^{2}$ \\ 1 Group of Astrophysics and Gravity Theory, Institute of Physics, UMCS, ul. Radziszewskiego 10, 20-031 Lublin, Poland \\ e-mail: marcingruszecki@wp.pl \\ 2 School of Mathematics and Statistics, University of St Andrews, St Andrews, Fife KY16 9SS, UK
}

Received 15 June 2007 / Accepted 6 May 2008

ABSTRACT

\begin{abstract}
Aims. We consider a model of a two-dimensional curved solar coronal slab and explore the excitation and attenuation of fast magnetoacoustic vertical oscillations. We include a dense photosphere-like layer into the physical system.

Methods. The time-dependent, ideal magnetohydrodynamic equations are solved numerically to determine the spatial and temporal signatures of the impulsively excited oscillations.

Results. The numerical results reveal that the inclusion of the dense photosphere-like layer has a significant influence on the wave period $(P)$ and attenuation time $(\tau)$. The wave characteristics exhibit a stronger dependence on the mass density contrast between the loop and the photosphere than on the width of the transition layer, according to the parametric studies performed here. We find that $P$ decreases and $\tau / P$ grows with the mass density contrast between the photosphere-like layer and solar corona, $d_{\mathrm{ph}}$. At the limit of $d_{\mathrm{ph}} \rightarrow \infty, P$ and $\tau / P$ attain their values which correspond to the case when the photosphere-like layer is removed from the system and its action is mimicked by implementation of line-tying boundary conditions at the bottom boundary.

Conclusions. The inclusion of a dense photosphere-like layer results in more efficient excitation and attenuation of vertical waves oscillation, compared with the case of line-tying boundary conditions. The enhancement of the attenuation rate arises from energy leakage through the photosphere-like layer.
\end{abstract}

Key words. Sun: oscillations - Sun: corona - magnetohydrodynamics (MHD)

\section{Introduction}

Magnetic loops harbouring gas at temperatures in the range $1-$ $10 \mathrm{MK}$ are the main ingredients of the solar corona. These loops are able to sustain oscillations (Nakariakov et al. 1999; Aschwanden et al. 1999) that have been detected by the Transition Region and Coronal Explorer (TRACE) in cool loops (temperatures close to $1 \mathrm{MK}$ ) and by the Solar Ultraviolet Measurements of Emitted Radiation (SUMER) onboard SOHO in hot loops (temperatures greater than $6 \mathrm{MK}$ ). Hot loops were detected to oscillate mainly in a slow magnetoacoustic mode (Wang et al. 2002, 2005), while cool loops primarily sustain fast magnetoacoustic kink oscillations. The latter oscillations have been observed in two polarizations: Aschwanden et al. (1999), Nakariakov et al. (1999), Schrijver et al. (1999), Van Doorsselaere et al. (2007) and many others detected horizontal kink oscillations, while Wang \& Solanki (2004) observed vertical kink oscillations. An excellent review of more observational events can be found in Nakariakov \& Verwichte (2005). The detection and identification of magnetohydrodynamic (MHD) waves and oscillations in the solar corona from recent observations using ground-based and space-born instruments has made MHD coronal seismology a viable diagnostic tool for the determination of unknown parameters of the corona (Uchida 1970; Roberts et al. 1984).

Various kinds of oscillations in a curved loop were recently modelled by a number of authors (e.g., Smith et al. 1997; Brady \& Arber 2005; del Zanna et al. 2005; Murawski et al. 2005a,b; Selwa et al. 2005, 2006; Verwichte et al. 2006a; Díaz et al. 2006a; Gruszecki et al. 2006; Ofman 2007;
McLaughlin \& Ofman 2008). These authors considered several attenuation mechanisms of these oscillations. For instance, Smith et al. (1997) studied the leakage of fast sausage and kink oscillations with exponentially increasing Alfvén speed profile, and concluded that the rate of energy leakage is inversely proportional to the wave period. Brady \& Arber (2005) studied the leakage of fast kink oscillations, initially excited by footpoint driving. They explained the leakage as wave tunnelling through an evanescent barrier above the coronal loop. Van Doorsselaere et al. (2004) discussed a decay of loop oscillations. They considered the modification of the quasi-modes of a loop structure due to curvature of magnetic field lines. Here, the decay of loop oscillations was due to resonant coupling between the global kink oscillation and a local torsional Alfvén mode. It was found that curvature exerts only a small effect on the decay rate of modes in the loop but the authors restricted themselves to a linear Alfvén speed profile for which waves are trapped. Recently Verwichte et al. (2006a,b,c) developed analytical models for vertically polarised fast magnetoacoustic waves in a curved coronal loop. In Verwichte et al. (2006a) the coronal loop was modelled in the limit of cold plasma approximation as a curved magnetic slab for equilibrium density given by a piece-wise continuous power law profile. Depending on the value of the power law index, the wave modes were trapped or they were all subject to lateral wave leakage (upward or downward). Verwichte et al. (2006b) confirmed that vertically polarised fast magnetoacoustic kink oscillations of isolated coronal loops may be efficiently attenuated due to lateral leakage. They also showed that fast kink oscillations in slender loops may result in significant density perturbations. Verwichte et al. (2006c) concluded that the mechanism 
of lateral wave leakage was efficient in the attenuation of vertically polarised fast kink oscillations. However, the theoretical model predicted attenuation rates that were higher than observational data. Díaz et al. (2006a) showed how the normal fast magnetoacoustic modes of a coronal loop are modified by the addition of density structure along the loop axis. The main result was that the frequency and spatial structure of the trapped modes were very sensitive to density variations within the loop. The effect of a dense layer near the footpoints was dealt with by Díaz et al. (2004), McEwan et al. (2006), Erdélyi \& Verth (2007) and Dymova \& Ruderman (2007).

Of particular importance to this paper is the work of Selwa et al. (2006), who investigated impulsively excited fast magnetoacoustic oscillations and reported that wave periods and attenuation times of the excited waves depend on the position of a pulse below the loop summit, as well as on the width of the pulse. However, Selwa et al. (2006) utilised a constant background mass density field and the response of the photosphere was modelled by the implementation of line-tying boundary conditions, whereas the real solar atmosphere is highly stratified and the mass density field varies over several orders in magnitude.

The main aim of this paper is to extend the model of Selwa et al. (2006) by implementing a dense photosphere-like layer into the system. We do not include the effect of gravity in our system, and so we model the photosphere by a layer of uniform plasma. More realistic models with a gravity force will be considered in future studies. In particular, we explore the importance of the mass density ratio, profile and depth of a photospherelike layer and investigate the effect of different excitation mechanisms on the resulting vertical kink oscillations in our curved solar coronal slab.

This paper is organised as follows: The numerical model is described in Sect. 2. The numerical results are presented in Sect. 3 and several subsections explore the effect of the different parameters. The conclusions are given in Sect. 4.

\section{Numerical model}

Our model is comprised of a strongly magnetised plasma that is described by the ideal magnetohydrodynamic (MHD) equations for the adiabatic case:

$$
\begin{array}{r}
\frac{\partial \varrho}{\partial t}+\nabla \cdot(\varrho \boldsymbol{V})=0, \\
\varrho \frac{\partial \boldsymbol{V}}{\partial t}+\varrho(\boldsymbol{V} \cdot \nabla) \boldsymbol{V}=-\nabla p+\frac{1}{\mu}(\nabla \times \boldsymbol{B}) \times \boldsymbol{B}, \\
\frac{\partial p}{\partial t}+\boldsymbol{V} \cdot \nabla p=-\gamma p \nabla \cdot \boldsymbol{V}, \\
\frac{\partial \boldsymbol{B}}{\partial t}=\nabla \times(\boldsymbol{V} \times \boldsymbol{B}), \\
\nabla \cdot \boldsymbol{B}=0, \\
p=\frac{R}{\hat{\mu}} \varrho T,
\end{array}
$$

where $\gamma=5 / 3$ is the adiabatic index, $\mu$ is the magnetic permeability, $\varrho$ is mass density, $\boldsymbol{V}$ is flow velocity, $p$ is gas pressure, $\boldsymbol{B}$ is the magnetic field, $T$ is temperature and $\hat{\mu}$ is the mean atomic weight (average mass per particle in units of mass of a proton). In the following work, we neglect gravity, thermal conduction, radiation, plasma heating, viscosity and resistivity.

\subsection{Equilibrium configuration}

We implement the coronal arcade model that was recently considered by Gruszecki et al. (2006) in their study of vertical oscillations of multi-stranded loop structures. Here, the equilibrium magnetic field components are expressed with the magnetic potential $A_{\mathrm{e}}$, where $\boldsymbol{B}_{\mathrm{e}}=\nabla \times\left(A_{\mathrm{e}} \hat{\boldsymbol{y}}\right)$ :

$A_{\mathrm{e}}(x, z)=B_{0} \Lambda_{B} \cos \left(x / \Lambda_{B}\right) \exp \left(-z / \Lambda_{B}\right)$,

where $B_{0}$ is the magnetic field at $z=0$ and $\Lambda_{B}$ is the magnetic scale-height:

$\Lambda_{B}=\frac{2 L}{\pi}$

and $L$ is the horizontal half-width of the arcade. We set $L=$ $100 \mathrm{Mm}$ in this paper. Thus, our equilibrium magnetic field is given by:

$\boldsymbol{B}_{\mathrm{e}}=B_{0}\left[\cos \left(x / \Lambda_{B}\right), 0,-\sin \left(x / \Lambda_{B}\right)\right] \exp \left(-z / \Lambda_{B}\right)$.

For a detailed discussion of the above equations see Priest (1982).

In our model, the coronal arcade is placed in a twodimensional and initially motionless environment $\left(\boldsymbol{V}_{\mathrm{e}}=0\right)$. In addition, the equilibrium magnetic field is current free, $\nabla \times \boldsymbol{B}_{\mathrm{e}}=$ 0 , and so from Eq. (2) we see that equilibrium $p_{\mathrm{e}}=$ const. $=p_{0}$. Figure 1 (top panel) shows initial profiles of mass density, gas pressure and temperature drawn along the line $x / L=0$.

To model our coronal loop, we consider a curved slab which is embedded in the arcade in such a way that its centre follows a specific magnetic field line. The slab is established by the following mass density profile:

$\varrho(x, z)=\xi \varrho_{0} \exp \left\{-\frac{\left[z-z_{\mathrm{c}}(x)\right]^{2}}{\sigma(x, z)^{2}}\right\}$.

Here $\sigma(x, z)$ is a half-width of the slab and $\xi$ denotes the mass density contrast between the slab and the ambient medium. In this paper, we set $\xi=10$ (as in Selwa et al. 2006). The symbol $z_{\mathrm{c}}(x)$ corresponds to a magnetic field line of our choice where $A_{\mathrm{e}}(x, z)=$ const. We choose $z_{\mathrm{c}}(x)$ with the use of the following formula:

$z_{\mathrm{c}}(x)=\frac{1}{2} \Lambda_{B} \log \left[\frac{\cos \left(x_{1} / \Lambda_{B}\right)}{\cos \left(x / \Lambda_{B}\right)} \cdot \frac{\cos \left(x_{2} / \Lambda_{B}\right)}{\cos \left(x / \Lambda_{B}\right)}\right]$,

where $x_{1}=-0.7 L$ and $x_{2}=x_{1}+\sigma\left(x_{1}, 0\right)$. The symbol $x_{1}$ corresponds to the external left slab footpoint. Here $\sigma\left(x_{1}, 0\right)=$ $2.5 \mathrm{Mm}$ is the width of the slab at $z=0$. Note that for this choice of parameters, the curved slab does not have a circular shape: the average radius and length are $\sim 70 \mathrm{Mm}$ and $\sim 190 \mathrm{Mm}$, respectively. These values are close to the observational data of Wang \& Solanki (2004).

In this paper, we extend the work of Selwa et al. (2006) by implementing a dense photosphere-like layer. This layer is modeled by:

$\varrho_{\mathrm{e}}(z)=\varrho_{0}+\frac{1}{2} \varrho_{0}\left(d_{\mathrm{ph}}-1\right)\left[1-\tanh \left(\frac{z}{s_{\mathrm{ph}}}\right)\right]$,

where $d_{\mathrm{ph}}$ is the ratio of the mass density of the photosphere to the ambient medium, and $s_{\mathrm{ph}}$ the half-width of the tanh profile. Hence, $s_{\mathrm{ph}}$ is related to the gradient of the mass density profile and is a measure of the "smoothness" of the profile. In our simulations, we choose $\varrho_{0}=10^{-15} \mathrm{~kg} \mathrm{~m}^{-3}$. Thus, within our photosphere the plasma is compressed to a mass density of $\left(\varrho_{0} \times d_{\mathrm{ph}}\right)$. 

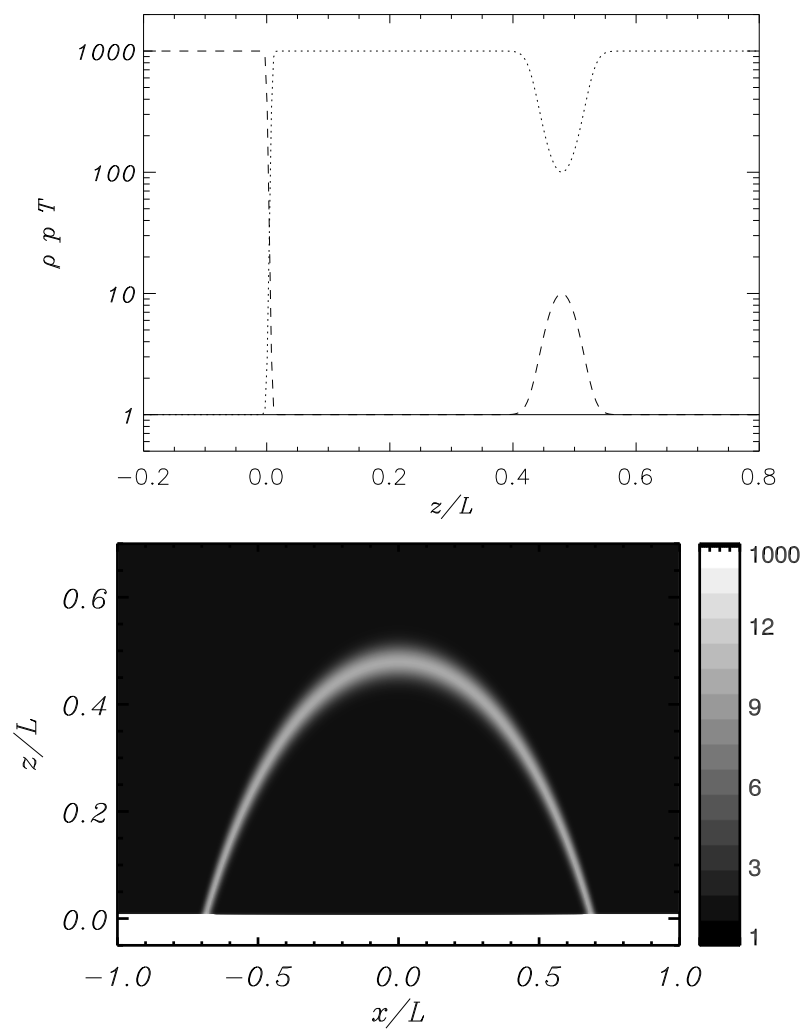

Fig. 1. (Top) Equilibrium configuration of mass density (dashed line), gas pressure (solid line) and temperature (dotted line) is measured in units (gas pressure in $2.68 \times 10^{-8} \mathrm{~Pa}$ and temperature in $T=2 \times 10^{6} \mathrm{~K}$ ) collected along $x / L=0$ at $t=0 \mathrm{~s}$. (Bottom) Equilibrium mass density profile (grey scale: $10^{-15} \mathrm{~kg} \mathrm{~m}^{-3}$ ) in the $x-z$ plane at $t=0 \mathrm{~s}$. The dense photosphere-like layer is located below the curved slab Note that only a part of the simulation region is displayed $(z \in[-0.5 L, 2 L])$.

Figure 1 (top and bottom panels) shows the initial mass density configuration of the arcade (where we have taken $d_{\mathrm{ph}}=10^{3}$ ).

In this paper, we choose $c_{\mathrm{A}}=10^{6} \mathrm{~m} \mathrm{~s}^{-1}$ for the Alfvén speed and $c_{\mathrm{s}}=2 \times 10^{5} \mathrm{~m} \mathrm{~s}^{-1}$ for the sound speed. Thus, plasma beta $\beta=$ $2 c_{\mathrm{s}}^{2} / \gamma c_{\mathrm{A}}^{2}=0.012$ at the reference level $z / L=0$. Gas pressure $p_{0}$ and magnetic field $B_{0}$ at $z / L=0$ can also be evaluated from the following expressions:

$p_{0}=\frac{c_{\mathrm{s}}^{2} \varrho_{0}}{\gamma}, \quad B_{0}=\sqrt{\frac{2 \mu p_{0}}{\beta}}$.

\section{Numerical results}

We solve numerically Eqs. (1)-(5) using the numerical code Athena as detailed in Gardiner \& Stone (2005). This code implements the higher-order Godunov method and solves the timedependent, ideal MHD equations. The algorithm is single step, second-order accurate in space and time, and is based on the piecewise parabolic method (e.g. Woodward \& Collela 1984). The numerical scheme is supported by a constrained transport scheme to keep the divergence-free constraint on the magnetic field (Eq. (5)).

We consider an Eulerian grid with the $x$ - and $z$-dimensions $[-L, L] \times[-0.5 L, 2 L]$. The box is covered by a uniform grid of $450 \times 630$ numerical cells. We performed grid convergence studies to show that the numerical results are not affected by insufficient spatial resolution. We implement open boundary conditions for all perturbed plasma quantities at all boundaries of the numerical box. These conditions were implemented by requiring that at all boundaries $\partial \delta f / \partial r=0$, where $\delta f$ denotes a perturbed plasma quantity and $r$ is a spatial coordinate that is perpendicular to the boundary. For such a choice of the boundary conditions fast magnetoacoustic waves essentially freely leave the simulation region. Some small reflections occur only from the top corners of the simulation region.

\subsection{Initial excitation through pressure pulse}

In this paper, our interest is in impulsively excited fast magnetoacoustic waves, and we will consider impulsive excitation using a gas pressure pulse (Selwa et al. 2006).

Here, a gas pressure pulse takes the form:

$p=p_{\mathrm{e}}\left[1+A_{\mathrm{p}} \exp \left(-\frac{\left(x-x_{0}\right)^{2}+\left(z-z_{0}\right)^{2}}{w^{2}}\right)\right]$,

where $A_{\mathrm{p}}$ is a relative amplitude of the initial pulse, $w$ is its width and $z_{0}$ is the initial height from which we launch the pressure pulse (here $z_{0}=0$ ). To keep symmetry in the $x$-direction we fix $x_{0}=0$.

We begin our studies by choosing a gas pressure pulse with amplitude $A_{\mathrm{p}}=5$ and width $w=0.35 \mathrm{~L}$. For this choice we obtain the maximum shift similar to that observed by Wang \& Solanki (2004). All other parameters are the same as those used by Selwa et al. (2006). In addition, we choose and hold fixed the mass density contrast between the curved slab and the ambient medium at $\xi=10$, and choose the width of the photospherelike layer to be $s_{\mathrm{ph}}=0.025 \mathrm{~L}$. Note that in physical units, our choice of $L=100 \mathrm{Mm}$ corresponds to $s_{\mathrm{ph}}=2.5 \mathrm{Mm}$, which is slightly larger than the sum of the heights of the photosphere and chromosphere.

The curved slab starts to oscillate when the fast magnetoacoustic waves that result from the initial pulse reach the slab. Figure 2 displays the time-signature of the mass density that is collected in time at the slab apex (along $x / L=0$ ). Note that these oscillations decay very fast in time and the slab displays an offset, i.e. it does not return to its initial position. In addition, this offset is larger than that in the case where the photospherelike layer is absent (in comparison with Selwa et al. 2006). Note that Selwa et al. (2006) did not consider a photosphere-like layer and instead implemented line-tying boundary conditions at the bottom boundary (the line-tying boundary conditions fix the velocity to $\boldsymbol{V}=0$ ). Whilst the line-tying boundary conditions lead to more wave reflection at $z=0$, the presence of a dense photosphere-like layer in our system results in a partial wave reflection and partial penetration of waves into this layer.

To better understand the phenomenon of the offset, we return to Eq. (14) and consider a range of amplitude, $A_{\mathrm{p}}$. By varying $A_{\mathrm{p}}$, we vary the amount of energy which is deposited between the slab and the photosphere-like layer. When the amount of the deposited energy is larger, the offset should also increase. We find that the offset grows linearly with $A_{\mathrm{p}}$ reaching values between 2 and $8 \mathrm{Mm}$ for $A_{\mathrm{p}}$ in the range 2 to $7 p_{\mathrm{e}}$. For a smaller amplitude pulse we approach the limit of a linear case for which the offset disappears (Verwichte et al. 2006b; Díaz et al. 2006a). A larger amplitude pulse introduces more non-linear effects which result in a larger offset (Selwa et al. 2006). We conclude that the offset does indeed result from energy being deposited in the region below the slab apex.

To estimate wave period $P$ and attenuation time $\tau$, we remove a trend from the time-signature of Fig. 2. To achieve this we find the position of the centre of the slab, $z(t)$, and fit this with a log 


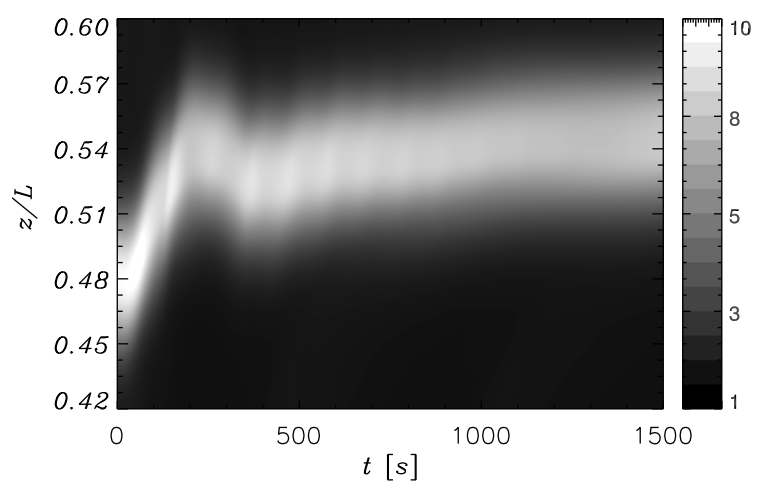

Fig. 2. Time-signature of mass density collected at the curved slab apex (along $x / L=0$ line). At $t=0$, the slab is 10 times denser than the ambient medium: $\xi=10$ (grey scale: $10^{-15} \mathrm{~kg} \mathrm{~m}^{-3}$ ).

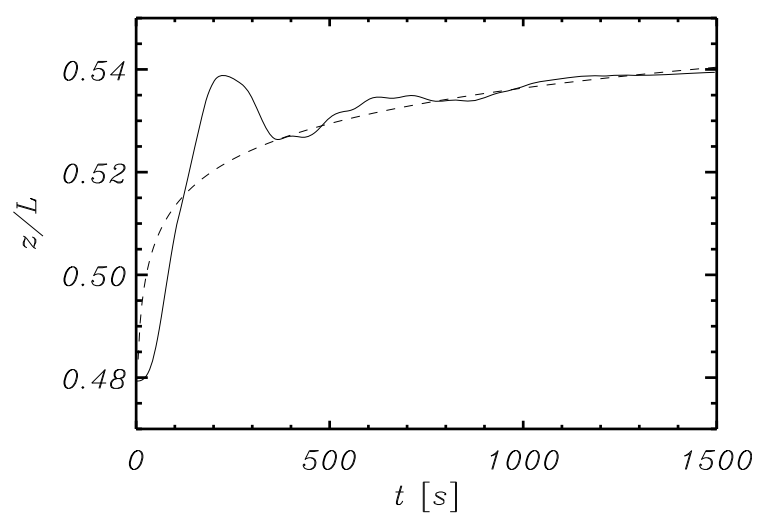

Fig. 3. Solid line denotes $z(t)$, which is the position of the centre of the curved slab in the time-signature of Fig. 2. Dashed line denotes a fitted logarithmic trend, $z_{\text {trend }}(t)$.

function $z_{\text {trend }}(t)$. This can be seen in Fig. 3. Values of $P$ and $\tau$ are obtained by fitting the attenuated sine function to $z_{0}(t)=z(t)-$ $z_{\text {trend }}(t)$, as detailed in Gruszecki et al. (2006). This estimation was made for all cases when the mass density contrast between photosphere-like layer and corona, $d_{\text {ph }}$, was smaller than 5000 . For a higher value of $d_{\mathrm{ph}}$ we can fit an attenuated sine function to the signal $z(t)$, because the offset is significantly smaller and we do not need to filter out $z_{\text {trend }}(t)$.

\subsection{Photospheric mass density contrast $d_{\mathrm{ph}}$}

We now investigate the importance of the photosphere mass density contrast, $d_{\mathrm{ph}}$, on the wave characteristics. From Fig. 4 (left panel) we see that the maximum vertical shift of the apex of the slab decreases with $d_{\mathrm{ph}}$. This is because for a larger value of $d_{\mathrm{ph}}$, the magnetic field lines are more strongly rooted in the dense photosphere-like layer. In addition, it is intuitive that for a larger value of $d_{\mathrm{ph}}$, the magnetic field lines are harder to stretch out from the photosphere-like layer (due to increased inertia). It is clear that for $d_{\mathrm{ph}}=100$, magnetic field lines are stretched more. It is known that longer loops exhibit longer wave period oscillations and, correspondingly, shorter loops exhibit shorter wave period oscillations. The smaller the value of $z_{\mathrm{m}}$, the less the slab becomes stretched out and, hence, the shorter the wave period oscillations will be. This phenomenon is seen in our simulations (Fig. 4, middle panel).

For a higher value of $d_{\mathrm{ph}}$, fewer magnetoacoustic waves leak to the photosphere-like layer (due to increased wave reflection). To confirm this, we estimate the time-evolution of the kinetic
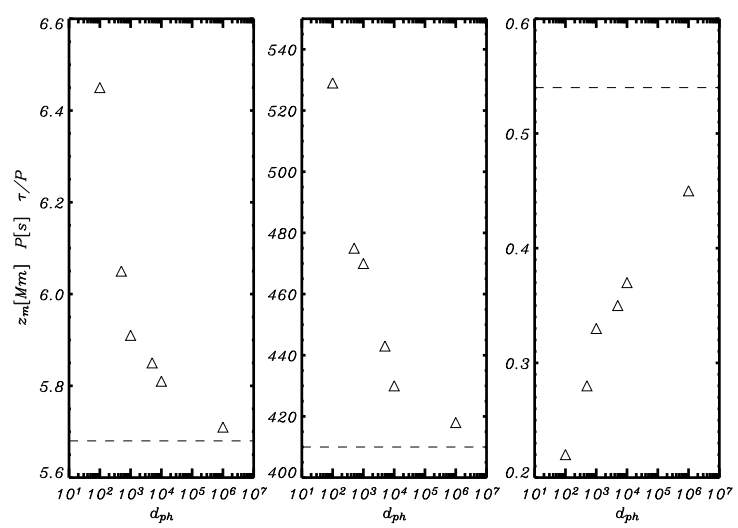

Fig. 4. (Left) Maximum of vertical shift of the curved slab apex $\left(z_{\mathrm{m}}\right)$ vs. mass density contrast between the photosphere-like layer and the ambient medium $\left(d_{\mathrm{ph}}\right)$. (Middle) Wave period $P$ vs. $d_{\mathrm{ph}}$. (Right) Ratio of attenuation time to wave period $(\tau / P)$ vs. $d_{\mathrm{ph}}$. Horizontal dashed lines correspond to the data that was obtained for line-tying boundary conditions at the bottom.

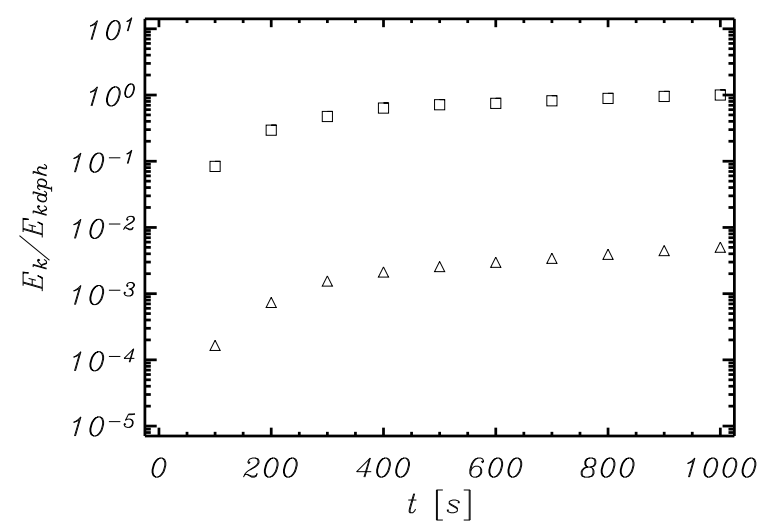

Fig. 5. Time-signatures of the normalized kinetic energy density of fast and slow magnetoacoustic waves in the photosphere-like layer, $E_{\mathrm{k}}(t) / E_{\mathrm{kd}}=100(t=1000 \mathrm{~s})$, for $d_{\mathrm{ph}}=100$ (squares) and $d_{\mathrm{ph}}=1000$ (triangles). At $t=1000 \mathrm{~s} E_{\mathrm{k}}=1.8 \times 10^{-11} \mathrm{~J} \mathrm{~m}^{-3}$ for $d_{\mathrm{ph}}=100$.

energy of magnetoacoustic waves within the photosphere-like layer (Fig. 5). From the obtained results we infer that more energy leaks, i.e. penetrates, into the photosphere-like layer for the case of $d_{\mathrm{ph}}=100$ than for $d_{\mathrm{ph}}=1000$. Thus, oscillations within a curved slab should be more attenuated for a lower value of $d_{\mathrm{ph}}$. This behaviour holds over a range of $d_{\mathrm{ph}}$ values (Fig. 4, right panel).

In Fig. 4 horizontal dashed lines correspond to the data that was obtained for line-tying boundary conditions implemented without any presence of the photosphere-like layer. From these results we infer that in the limit of $d_{\mathrm{ph}} \rightarrow \infty$ values of $z_{\mathrm{m}}, P$ and $\tau / P$ tend to the line-tying boundary conditions data. Thus, we conclude that the presence of the dense photosphere-like layer exerts a significant influence on time-signatures of vertical loop oscillations.

From Fig. 6 which displays mass density profiles for $d_{\mathrm{ph}}=$ 100 (top panel) and $d_{\mathrm{ph}}=1000$ (bottom panel) we infer that the photosphere-like layer becomes modified by the magnetoacoustic waves which enter this layer. The magnitude of this modification depends on $d_{\mathrm{ph}}$ and is, as expected, weaker for a larger value of $d_{\mathrm{ph}}$. 

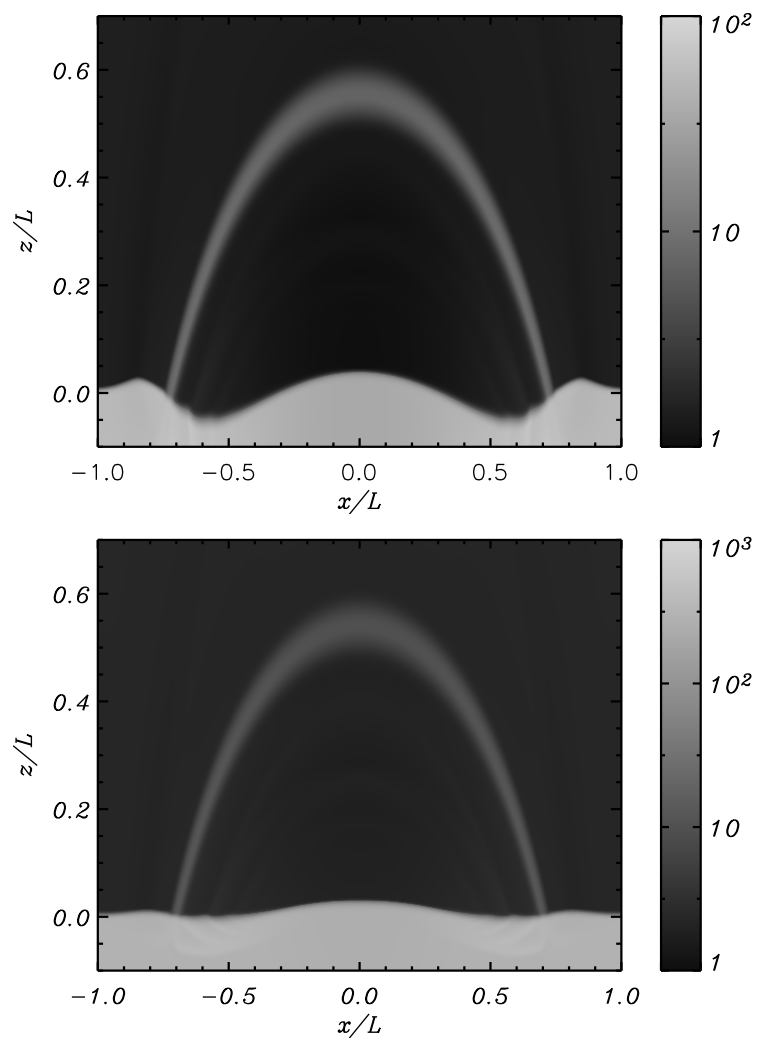

Fig. 6. Mass density profile at $t=1200 \mathrm{~s}$ for (top) $d_{\mathrm{ph}}=100$ and (bottom) $d_{\mathrm{ph}}=1000$ (grey scale: $10^{-15} \mathrm{~kg} \mathrm{~m}^{-3}$ ).

\subsection{Smoothness $\mathrm{s}_{\mathrm{ph}}$ of the photosphere-like layer}

We now consider the importance of the width $s_{\mathrm{ph}}$ of the photosphere-like layer on wave characteristics, where $s_{\text {ph }}$ is related to the gradient or steepness of the mass density profile (see Eq. (12)). For the following simulations, we fix $d_{\text {ph }}=1000$.

We would expect that for a smoother mass density profile (larger values of $s_{\mathrm{ph}}$ ) the magnetic field lines would be less strongly rooted within the photosphere-like layer. In this case, the magnetic field lines would be stretched out further from the photosphere and, consequently, we would expect larger values of $z_{\mathrm{m}}$. This behaviour can be seen in Table 1 , but we note that the effect of $s_{\mathrm{ph}}$ on $z_{\mathrm{m}}$ is small, i.e. for $0.25 \mathrm{Mm} \leq s_{\mathrm{ph}} \leq 3 \mathrm{Mm}$ the apex is only shifted up in the range $5.95 \mathrm{Mm} \leq z_{\max } \leq 5.99 \mathrm{Mm}$.

Table 1 also shows that wave period $P$ grows with $s_{\mathrm{ph}}$, whereas the ratio $\tau / P$ declines for larger values of $s_{\mathrm{ph}}$. As explained above, for a larger value of $s_{\mathrm{ph}}$, the slab is less rooted in the photosphere-like layer and hence becomes more stretched by the initial pulse (as seen in Table 1). Values from Table 1 agree with our former claims that longer slabs exhibit longer wave periods $P$. Previous studies of 2D curved slabs revealed that if energy leakage to the ambient corona is responsible for wave attenuation then short wave period oscillations experience less attenuation than long oscillations (see, for example, Selwa et al. 2006; Gruszecki et al. 2006; Verwichte et al. 2006b). This phenomenon is also seen in our simulations (Table 1).

\section{Conclusions}

We have developed a two-dimensional model of a curved coronal slab that is anchored in a dense photosphere-like layer. Vertical fast magnetoacoustic kink oscillations were triggered by an initial gas pressure pulse launched underneath a curved slab.
Table 1. Maximum of vertical shift of the curved slab apex $z_{\mathrm{m}}$, wave period $P$ and the ratio $\tau / P$ for four values of smoothness $s_{\mathrm{ph}}$ of the photosphere-like layer.

\begin{tabular}{cccc}
\hline \hline$s_{\mathrm{ph}}[\mathrm{Mm}]$ & $z_{\mathrm{m}}[\mathrm{Mm}]$ & $P[\mathrm{~s}]$ & $\tau / P$ \\
\hline 0.25 & 5.95 & 436 & 0.328 \\
1.0 & 5.958 & 442 & 0.325 \\
2.0 & 5.972 & 457 & 0.305 \\
3.0 & 5.987 & 477 & 0.272 \\
\hline
\end{tabular}

Such a pulse excited magnetoacoustic waves among which fast kink waves dominated.

Our numerical results show that varying the density contrast $d_{\mathrm{ph}}$ and smoothness $s_{\mathrm{ph}}$ of the dense photosphere-like layer varies the time-signatures of the resulting oscillations, specifically that decreasing the density contrast $d_{\mathrm{ph}}$ increases the maximum vertical shift, i.e. offset, of the loop, and that increasing the smoothness $s_{\text {ph }}$, also increases the offset. This is because increasing $d_{\mathrm{ph}}$ and decreasing $s_{\mathrm{ph}}$ correspond to the magnetic fieldlines being more strongly rooted in the photosphere, and hence they are harder to stretch. We find that the oscillations have a strong dependence on these parameters specifically because the inclusion of a photosphere-like layer affects the leakage of the fast magnetoacoustic waves into the photosphere region and influences the energy transfer during the initial transient stage. It is interesting that the observed attenuation of the oscillations is not associated with any non-ideal effect which would result in the dissipation of eigenmodes, but instead is related to the transference of the impulsive excitation to the eigenmode solution and energy leakage from the curved slab to the ambient medium.

In our model, the wave periods and attenuation times have a stronger dependence on the photosphere mass density contrast $\left(d_{\mathrm{ph}}\right)$ than on the width parameter $\left(s_{\mathrm{ph}}\right)$. Our results reveal that for a sufficiently large value of $d_{\mathrm{ph}}$, wave period $P$ and $\tau / P$ are close to the results obtained for the case of implementing line-tying boundary conditions. For a larger value of $d_{\mathrm{ph}}$, energy leakage into the photosphere-like layer is limited and as a result the slab absorbs more energy.

The numerical results obtained in this paper correspond to the observed values for vertical oscillations; Wang \& Solanki (2004) record $z_{\mathrm{m}}=7.9 \mathrm{Mm}, P=234 \mathrm{~s}$ and $\tau / P \simeq 3$. In our simulations, the values of $z_{\mathrm{m}}$ are close to the observational data. Secondly, we found wave periods in the range 300-500 s which is approximately twice as large as the observational data, although the wave periods can be reduced by reducing the pulse width or amplitude (as found by Selwa et al. 2006). Finally, the average value of $\tau / P$ obtained from our simulations is about 0.4 . This value is about 6 times smaller than the observational data, suggesting that in our model, the wave attenuation is much too strong. Verwichte et al. (2006b) believed that the ratio $\tau / P \simeq 3$ is rather large and a ratio $\tau / P$ about three and half times smaller may be consistent with the observational signature of Wang \& Solanki (2004). If they are right, our ratio $\tau / P$ is more suitable. However, the observation of Wang \& Solanki (2004) is the only existing observation of a vertically polarized fast kink oscillation and for exact estimation of $P$ or $\tau / P$ more observational data is required.

Acknowledgements. M.G. thanks Profs. Valery Nakariakov and Bernard Roberts and Drs. Tony Arber and Erwin Verwichte for stimulating discussions. A part of this work was done during M.G.'s visit to the Universities of St Andrews and Warwick. K.M. is indebted to Tom Gardiner for his comments on numerical issues. The authors thank the referee for his/her stimulating comments. M.G.'s \& K.M.'s work was supported by a grant from the State Committee for Scientific 
Republic of Poland, with MNiI grant for years 2007-2010. J.A.M. acknowledges financial assistance from the St Andrews STFC Rolling Grant and from the Leverhulme Trust. The magnetohydrodynamic code used in this study was developed at Princeton University by Tom Gardiner, Jim Stone, Peter Teuben and John Hawley with support of the NSF Information Technology Research (ITR) program.

\section{References}

Aschwanden, M. J., Fletcher, L., Schrijver, C. J., \& Alexander, D. 1999, ApJ, 520,880

Brady, C. S., \& Arber, T. D. 2005, A\&A, 438, 733

Del Zanna, L., Schaekens, E., \& Velli, M. 2005, A\&A, 431, 1095

Díaz, A. J., Oliver, R., \& Ballester, J. L. 2004, ESA SP-547, 489

Díaz, A. J., Oliver, R., \& Ballester, J. L. 2006a, ApJ, 645, 766

Díaz, A. J., Zaqarashvili, T., \& Roberts, B. 2006b, A\&A, 455, 709

Dymova, M. V., \& Ruderman, M. S. 2007, A\&A, 463, 759

Erdélyi, R., \& Verth, G. 2007, A\&A, 462, 743

Gardiner, T. A., \& Stone, J. M. 2005, J. Comput. Phys., 205, 509

Gruszecki, M., Murawski, K., Selwa, M., \& Ofman, L. 2006, A\&A, 460, 887

McLaughlin, J. A., \& Ofman, L. 2008, ApJ, 682, 1338

McEwan, M. P., Donnelly, G. R., Díaz, A. J., \& Roberts, B. 2006, A\&A, 460, 893
Murawski, K., Selwa, M., \& Rossmanith, J. A. 2005a, Sol. Phys., 231, 87

Murawski, K., Selwa, M., \& Nocera, L. 2005b, A\&A, 437, 687

Nakariakov, V. M., \& Verwichte, E. 2005, Living Rev. Sol. Phys., 2, 3

Nakariakov, V. M., Ofman, L., Deluca, E. E., Roberts, B., \& Davila, J. M. 1999, Science, 285,862

Ofman, L. 2007, ApJ, 655, 1134

Priest, E. R. 1982, Solar Magnetohydrodynamics (D. Reidel)

Schrijver, C. J., Title, A. M., Berger, T. E., et al. 1999, Sol. Phys., 187, 261

Roberts, B., Edwin, P. M., \& Benz, A. O. 1984, ApJ, 279, 857

Selwa, M., Murawski, K., Solanki, S. K., Wang, T. J., \& Tóth, G. 2005, A\&A, 440,385

Selwa, M., Solanki, S. K., Murawski, K., Wang, T. J., \& Shumlak, U. 2006, A\&A, 454, 653

Smith, J. M., Roberts, B., \& Oliver, R. 1997, A\&A, 327, 377

Uchida, Y. 1970, PASJ, 22, 341

Van Doorsselaere, T., Debosscher, A., \& Andries, J. 2004, A\&A, 424, 1065

Van Doorsselaere, T., Nakariakov, V. M., \& Verwichte, E. 2007, A\&A, 1051, 6361

Verwichte, E., Foullon, C., \& Nakariakov, V. M. 2006a, A\&A, 446, 1139

Verwichte, E., Foullon, C., \& Nakariakov, V. M. 2006b, A\&A, 449, 769

Verwichte, E., Foullon, C., \& Nakariakov, V. M. 2006c, A\&A, 452, 615

Wang, T. J., \& Solanki, S. K. 2004, A\&A, 421, L33

Woodward, P. R., \& Colella, P. 1984, J. Comput. Phys., 54, 174 